\title{
PAROXYSMAL NODAL TACHYCARDIA WITH RETROGRADE HEART BLOCK
}

\author{
BY \\ J. A. $\mathrm{COSH}$ \\ From the Department of Medicine, University of Bristol \\ Received February 14,1950
}

Paroxysmal nodal tachycardia is not uncommon, and may be divided into three types, in which the rhythm arises in the upper, middle, or lower part of the A-V node. Low nodal tachycardia, which is characterized by an inverted $P$ wave following the $R$ wave at a constant interval, was found in 18 out of 253, and 8 out of 42 cases of paroxysmal tachycardia analysed by the electrocardiograph in the series reported by Hume (1930) and Campbell and Elliott (1939) respectively, i.e. in 9 per cent.

Lewis (1925) showed that in "low nodal " rhythm produced experimentally, depression of conduction, such as follows vagal stimulation, leads to gradual lengthening of the R-P interval until auricular beats may be missed. It is rare to find this phenomenon clinically, and the case of Drury (1924) appears to be the only report of retrograde heart block during a paroxysm of tachycardia. For this reason, the following case is recorded. Other points of interest were also found.

\section{CASe RePORT}

The patient was a well-built girl of 11 years. Her past history was one of good health, apart from one illness which was probably her first attack. For this she was admitted to another hospital in February, 1949, with cough, nausea, and a regular pulse rate of 160 a minute. She had become unconscious a week before and this was probably the onset of the tachycardia. On admission she was not distressed; there was enlargement of the heart, and of the liver, but no congestion of the neck veins or œdema. Her tachycardia continued at the same rate until the fourth day in hospital, when the pulse rate gradually fell to 80 a minute. The heart returned to normal size within a few days. No electrocardiogram was taken during the tachycardia, but tracings were normal 11 and 24 days after it had stopped. It seems likely that this was her first paroxysm of tachycardia; it was certainly of 3 days and possibly of 10 days duration.

In April and May, 1949, when seen as an out-patient, she was well, and no abnormality was found in the heart, which was of normal size radiologically.

Present attack. On July 29, 1949, she complained of nausea, anorexia, and dizziness for no apparent cause, but was not conscious then, or at any time, of palpitation. She remained at home, but not in bed, and her symptoms continued. On August 4, she vomited once, and her doctor found a very rapid regular pulse rate, and sent her to hospital that day. On admission she was of healthy appearance and colour, and not distressed on lying flat, though she had some dyspnœa on effort. There was no venous congestion or œdema, and the liver could not be felt. The pulse was regular at about 200 a minute, and a corresponding pulsation was seen in the neck. The heart was enlarged, the apex beat being just beyond the mid-clavicular line in the fifth intercostal space. The heart sounds were normal and there were no murmurs. B.P. 100/80. No other abnormality was found. The pulse rate was not influenced by holding the breath, by changes of posture, or by pressure on either carotid sinus or on the eyeballs. These manœuvres had no effect clinically on any occasion. 


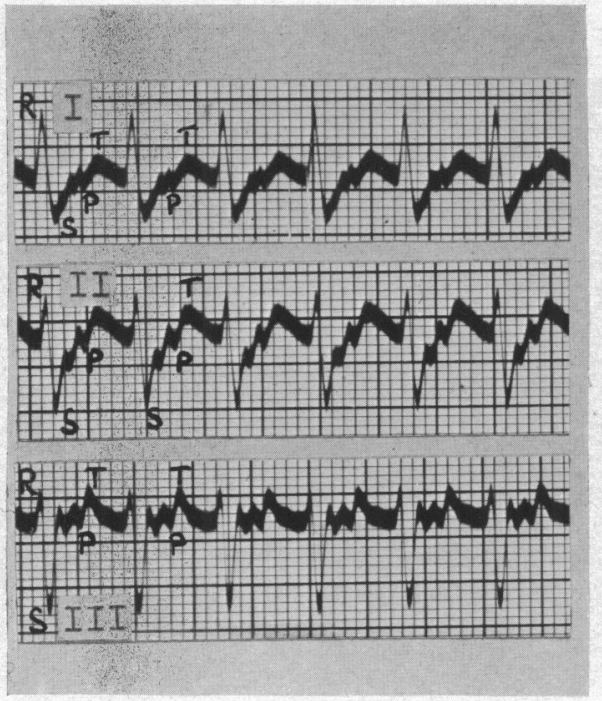

FIG. 1

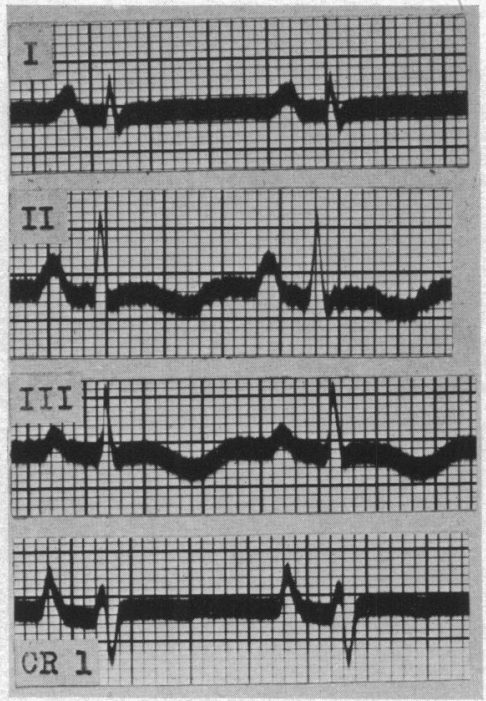

FIG. 2

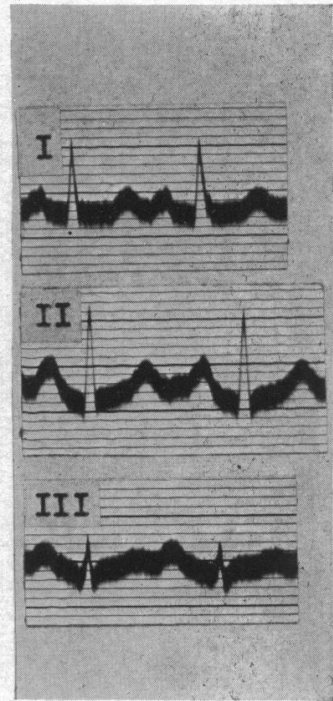

FIG. 3

FIG. 1.-4/8/49. Regular nodal tachycardia, rate 195 a minute. QRS duration $0 \cdot 12$ sec. R-P interval $0 \cdot 14$ sec. Fig. 2.-9/8/49. Sinus rhythm, rate 75 a minute. $\mathbf{P}$ waves are all upright, but $\mathrm{T}$ is flat or inverted in all and is broadened.

FIG. 3.-9/9/49. Sinus rhythm, rate 100 a minute. T waves now upright in all leads.

There was a regular tachycardia, rate 195 a minute. $P$ waves appeared to follow the ventricular complexes, and were inverted in leads II and III; the R-P interval was $0.14 \mathrm{sec}$; and the ventricular complexes were slightly abnormal in form and were of $0 \cdot 12 \mathrm{sec}$. duration (Fig. 1).

Carotid sinus pressure had no effect, but pressure on the eyeballs, while not changing the ventricular rate, produced progressive lengthening of the R-P intervals, and one auricular beat was missed (Fig. 4).

The patient was then given a trial dose of 3 grains of quinidine sulphate by mouth. On the following day (August 5) her tachycardia continued, with no change in the clinical findings, and she was comfortable apart from the anorexia and some nausea. A teleradiogram (Fig. 7A) showed generalized enlargement of the heart with a cardiothoracic ratio of 57 . There was no change in the limb leads, apart from a drop in the pulse rate to 178 a minute. Lead CR I showed pointed upright $P$ waves following the $R$ waves at a constant interval of $0.14 \mathrm{sec}$. (Fig. 5). Quinidine sulphate was given by mouth in divided doses, 8 grains on the 5th, 15 grains on the 6th, and 20 grains on August 7 and 8.

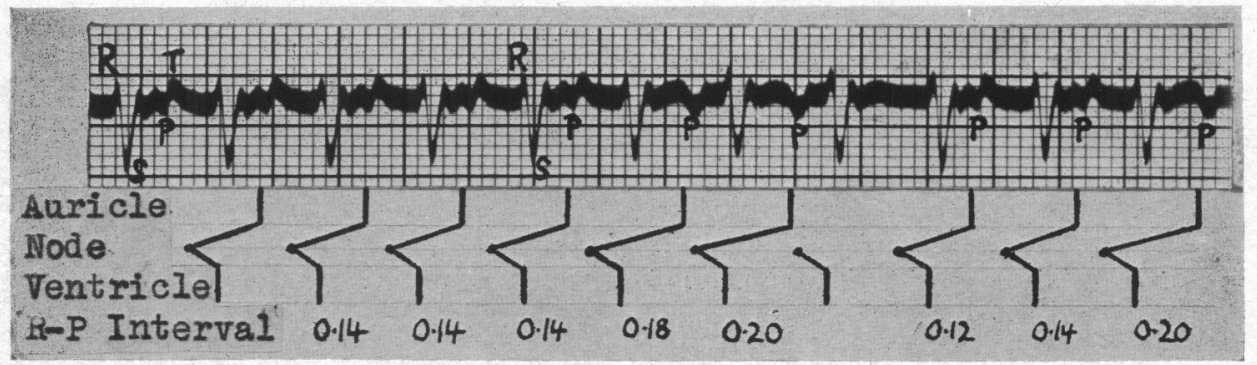

Fig. 4. $-4 / 8 / 49$. Lead III. Pressure on eyeballs commencing about $15 \mathrm{sec}$. before this tracing was taken produces block of retrograde spread of impulse to auricles without influencing ventricular rate. Probable mechanism is indicated. $\mathbf{R}-\mathbf{P}$ interval is prolonged and one auricular contraction is missed. 
On August 8, there was still no clinical change; the ventricular rate was 158 a minute in the limb leads, but was 170 a minute in the chest leads taken a few minutes later (Fig. 6). $P$ waves were unchanged in form, but occurred irregularly at a slower rate than the $R$ waves. In the strip of lead III seen here, 3:2 V-A block is constant, and in leads I and II a similar block predominates. In lead CR 1 the mechanism of a 3:2 retrograde block is tentatively illustrated, although it is possible that complete V-A dissociation had now arisen, as the auricular rate was nearly regular at 105 a minute, and the ventricular rate had accelerated a little. About 12 hours after these tracings were taken, the pulse was found to have slowed to less than 100 a minute, though the patient had noticed no change in her heart rate.

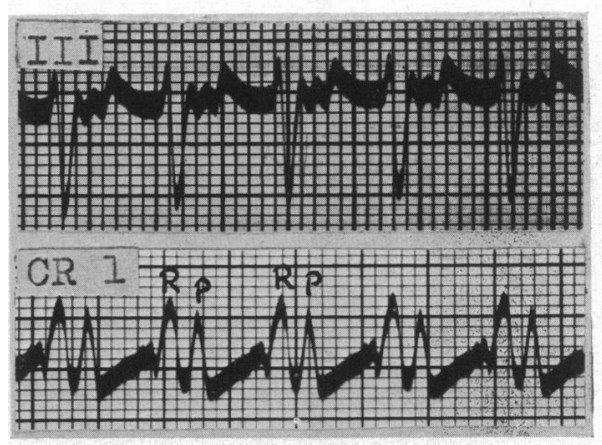

FIG. 5.-5/8/49. Limb leads as on previous day, but rate is now 178 a minute. Lead $C R 1$ shows $R$ wave followed by $P$ wave; compare with form of $P$ and $R$ in lead CR 1 in Fig. 6.
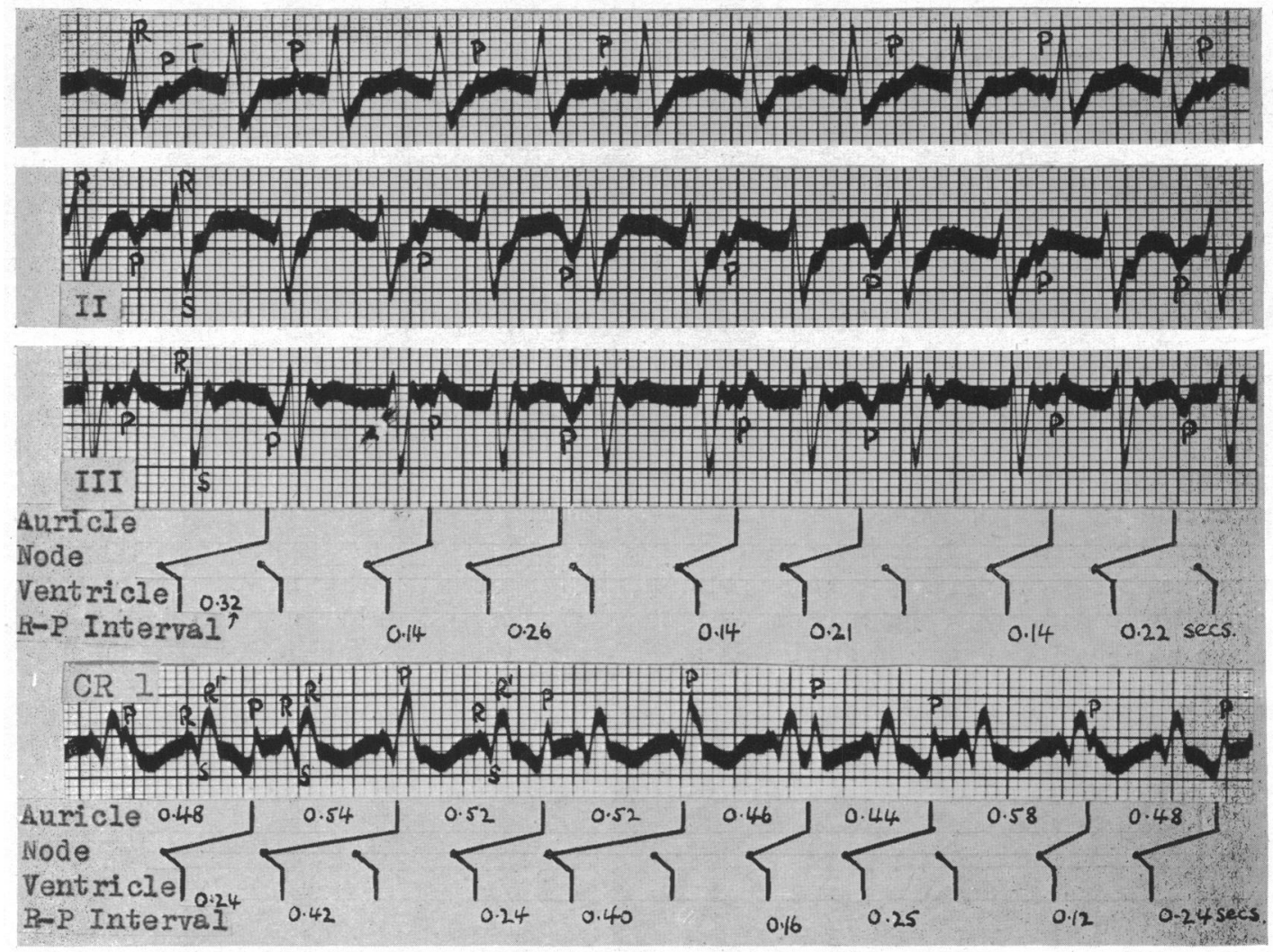

FIG. 6.-8/8/49. Regular ventricular tachycardia continues, rate 158 a minute in limb leads, 170 a minute in lead CR 1. P waves now occur at a slower rate than $R$ waves and 3:2 V-A block is seen in lead III and during most of leads I and II. A similar mechanism is suggested for leads CR 1. Note double $R$ wave is now more obvious in CR 1. QRS duration in all is 0.14 sec.

On the next day (August 9) the pulse was regular at 80 a minute; the patient felt better and had regained her appetite. Quinidine was stopped. There was regular sinus rhythm, rate 75 a minute, 
with inversion and broadening of T in leads II and III, as well as in leads CR 3 and 6, which are not shown here (Fig. 2). The time of electrical systole was prolonged by Bazett's formula, Q-T in lead II being $0.44 \mathrm{sec}$. for a cycle of $0.74 \mathrm{sec}$. This gives a value for factor $\mathrm{K}$ of 0.51 .

The patient was well subsequently, and normal rhythm was maintained. On the fourth day after return to normal, a teleradiogram (Fig. 7B) showed a normal heart size, with a cardiothoracic ratio of 49 per cent. On the following day the patient was discharged home.

When seen a month later, she was well, with a heart that appeared normal, and a sinus tachycardia of 100 a minute, slowing on carotid pressure, and with all $T$ waves upright (Fig. 3).

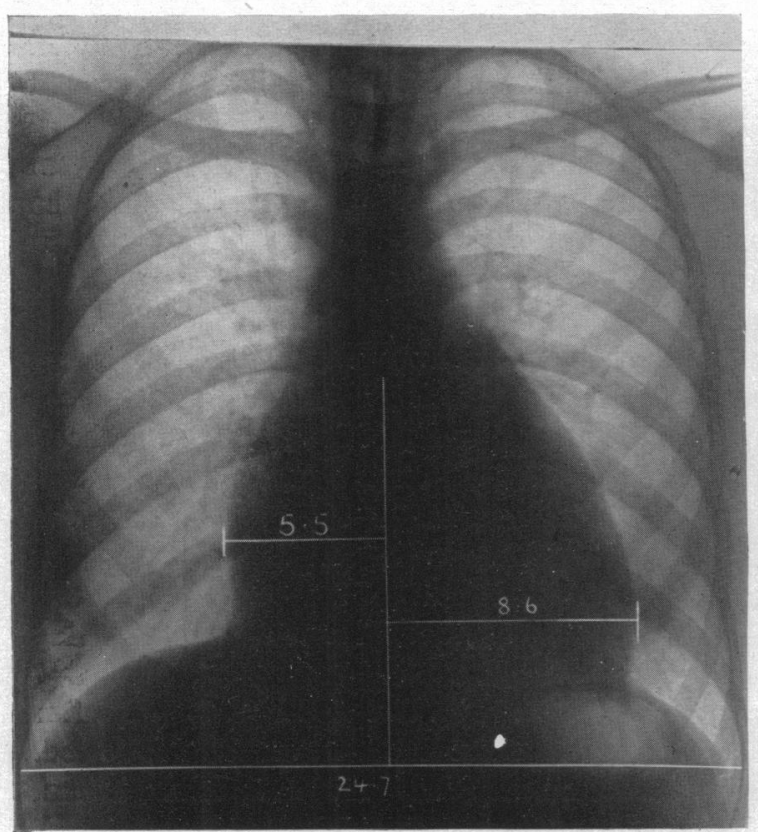

A

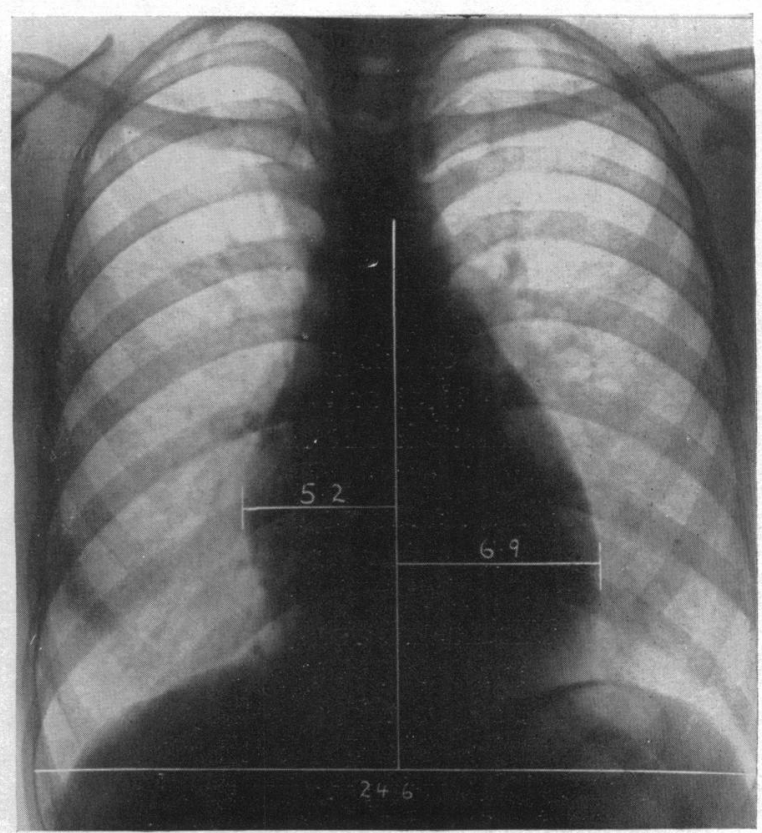

$\mathrm{B}$

FIG. 7.-(A) 5/8/49. Generalized enlargement of the heart on the eighth day of tachycardia. Cardiothoracic ratio 57. (B) $12 / 8 / 49$. Normal sized heart on the fourth day after return to normal rhythm. Cardiothoracic ratio 49 per cent.

Diagnosis. Low nodal tachycardia was diagnosed, as there was undoubted retrograde activation of the auricles, and vagal stimulation (Fig. 4) delayed this retrograde conduction in the way described by Lewis (1925). However, the ventricular complexes were not identical with those found afterwards, being slightly prolonged and deformed during the paroxysm. This difference was clearest when leads CR 1 were compared. Ventricular paroxysmal tachycardia could not be diagnosed, as, in that condition, when $P$ waves are seen, they occur at a slower rate and independently of the ventricles. Some degree of intra-ventricular block must therefore have occurred during the paroxysm.

\section{Discussion}

Retrograde heart block in nodal rhythm was first demonstrated by Lewis (1914); he found that vagal stimulation prolonged the R-P interval when this was seen in nodal rhythm that had been set up experimentally in dogs. Later, Lewis, White, and Meakins (1914) investigated in the cat the effect of asphyxia on nodal rhythm caused by cooling the S-A node. In 3 or 4 minutes of asphyxia, there was progressive lengthening of the R-P interval and finally 2:1 retrograde block of the 
auricle: they concluded that the part of the conducting system most susceptible to block lay at a higher level (i.e. nearer the auricle) than the actual seat of impulse discharge in the A-V node.

Clinical examples of similar block in nodal rhythm were given by White (1915) and by Gallavardin and Gravier (1921): in their cases the ventricular rate was of the order of 40-60 a minute, and vagal stimulation produced not only a marked slowing of the ventricle, but also a lengthening of the R-P interval; on one occasion, after digitalis, White found an R-P interval of 0.42 sec., which may be compared with that postulated in some beats in Fig. 6.

Drury (1924) described short paroxysms of nodal tachycardia occurring terminally in a man with infective endocarditis. In certain of his tracings he found progressive lengthening of the R-P interval; after this had reached about $0.14 \mathrm{sec}$., either an auricular beat was dropped or the impulse from the last auricular beat re-entered the ventricle and gave rise to a premature beat, i.e. reciprocal rhythm; this was found also by White, and by Gallavardin and Gravier, but was not seen in this case.

It is difficult to determine whether the retrograde block found on the last day of this paroxysm was induced by quinidine or not. In view of Drury's case (when no drug was given) and of the fact that reversion to normal rhythm occurred here shortly after retrograde block was found, it seems most likely that the block developed as a result of fatigue in the conducting system towards the end of a long paroxysm of tachycardia.

The duration of this paroxysm was probably ten days, but in neither attack was the patient conscious of the start or the finish of the tachycardia. Gradual slowing of the heart rate, from 195 to 158 a minute is unusual, as paroxysmal tachycardia has a typically constant rate in any one attack, although an individual may have different paroxysms at different rates (Campbell and Elliott, 1939). It seems unlikely that it was due to the quinidine given. A similar slowing, from 173 to 124 a minute in a seven-day paroxysm was described by Marvin (1923).

Cardiac dilatation was found during both paroxysms in this patient. On the 8 th day of this one, the cardiothoracic ratio was $\mathbf{0 . 5 7}$, instead of 0.45 and 0.49 on teleradiograms taken in May and September respectively.

Inversion and prolongation of T waves was seen in leads II, III, and CR 3 and 6 on the day after the paroxysm had stopped, but unfortunately the recession in these changes was not followed. Inversion of $T$ following paroxysmal tachycardia is well recognized (Campbell, 1942; Zimmerman, 1944), and the $T$ wave may be prolonged as well as inverted (Cossio et al., 1944; Ward, 1946), which change appears to be independent of the use of quinidine. In several illustrations in articles on this subject it will be seen that electrical systole (QT) is thus prolonged after a paroxysm of tachycardia. Using the formula of Bazett (1920) electrical systole was considerably prolonged, $\mathrm{K}$ being 0.51 in Fig. 2 instead of the normal 0.37 to 0.40 . This may have been due to residual cardiac dilatation after the paroxysm.

\section{SUMMARY}

A case of low nodal paroxysmal tachycardia is described, occurring in a girl of 11 years with an apparently normal heart. The paroxysm lasted probably ten days, and for the last four days the patient was treated with quinidine by mouth.

Reflex vagal stimulation on the seventh day of the tachycardia delayed retrograde conduction, prolonging the R-P interval progressively and causing an auricular beat to be dropped.

About 12 hours before the tachycardia stopped, spontaneous retrograde block was found to have developed, with predominantly 3:2 V-A rhythm.

During the period of observation the ventricular rate gradually slowed from 195 to 158 a minute.

Cardiac dilatation was found during the tachycardia, but the heart had returned to normal size on the fourth day after the paroxysm stopped.

Temporary inversion and broadening of $T$ waves was seen after the attack, causing lengthening of the time of electrical systole. 
My thanks are due to Dr. D. H. Davies for permission to publish this case and to him and Professor Bruce Perry for their helpful criticism.

\section{REFERENCES}

Bazett, H. C. (1920). Heart, 7, 353.

Campbell, M. (1942). Brit. Heart J., 4, 49.

-, and Elliott, G. A. (1939). Ibid, 1, 123.

Cossio, P., Vedoya, R., Berconsky,.I. Quoted in (1945) Amer. Heart J., 29, 650.

Drury, A. N. (1924). Heart, 11, 405.

Gallavardin, L., and Gravier, L. (1921). Arch. Mal. Caur, 14, 71.

Hume, W. E. (1930). Lancet 2, 1055.

Lewis, T. (1914). Ibid., 5, 247.

(1925). The Mechanism and Graphic Representation of the Heart Beat, Shaw, London.

- White, P. D., and Meakins, J. (1914). Heart, 5, 289.

Marvin, H. M. (1923). Ibid., 10, 279.

Ward, L. S. (1946). Amer. Heart J., 31, 645.

White, P. D. (1915). Arch. intern. Med., 16, 517.

Willius, F. A., and Barnes, A. R. (1924). Boston Med. Surg. J., 191, 666.

Zimmerman, S. L. (1944), J. Lab. Clin. Med., 29, 598. 